\title{
Analysis of the textured surface morphology of electrical discharge machining
}

\author{
Yang Yang1, a, Wenliang Qiü,b Dong Cai ${ }^{3, c}$ \\ 1,2,3School of mechanical and electrical engineering, vehicle engineering, East China Jiaotong \\ University. Jiangxi Province, Nanchang City shuanggan economic and Technological Development \\ Zone East Street, No. 808, 330013, China \\ a1292183825@qq.com, b913742626@qq.com, c1756379127@qq.com
}

\begin{abstract}
Keywords: Morphology; EDT; Optical Microscope; The average diameters of the pits; Abstract. The morphology of work piece surface obtained by electrical discharge texturing (EDT) is systemically analyzed in theory in this paper. By using negative electrode, graphite electrode and different electrical parameters (peak current, pulse width, pulse interval) experimental method to machining Non magnetic steel (20Mn23AlV), the characteristics of the morphology was studied and compared with each other under different electrical parameters. By using orthogonal experiment method and optical microscope, pits structure of the textured surface were observed and analyzed detailedly. The average diameters of the pits which play an important role to the study of surface light absorbing rate were measured and computed. Peak current has little influence on the average diameter of the pit, and the effect of the pulse width is larger.
\end{abstract}

\section{Introduction}

Compared with traditional machining, EDM is a kind of non-contact processing method, which uses high heat energy to remove the surface material of workpiece. Its characteristics are: high heat density between two electrodes, the discharge time is very short, the process is very complex ${ }^{[1]}$. In EDM process in general, the discharge time is usually controlled at tens of microseconds, in this moment, the electric energy will turn into heat, magnetic energy, kinetic energy, solar energy and other forms, and the most conducive energy to the material removal is heat making the surface material melt, vaporization and throw out the surface of the workpiece.

The workpiece surface achieved by EDM is composed of a number of concave-convex structures. These special structures have good optical properties. So some Optical phenomena will occur on the small pits of texturing surface as the external light radioed, just like reflection, refraction, diffraction, scattering and interference. Under the action of effective light absorption of textured surface, the loss of light reflection can be reduced when the light is radioing to EDT surface and enter into the small pits, so that more light will enter into the textured surface and multiple reflection will occurs in the internal of textured surface's pits. The light has the largest travel in the absorbing rate ${ }^{[2,3]}$, thereby it improving the rate of the light absorption on textured surface, reducing the reflected light and eventually the light returned to the medium. The factors that affect the micro morphology of the surface are mainly processing medium, electrical parameters, electrode material and the workpiece material. The processing medium, electrode material and workpiece material are consistent in the process of experiments, so the most important factor affecting the micro morphology is electrical parameters.

In terms of light-absorptivity of the surface, a series of theoretical experiments are carried out by many scholars from all over the world. However, the relationship among the discharge parameters and the shape of the pits and the light absorption rate of the pits is not deeply studied.

Abroad, From L. A. Dobranski and U. Gangopadhyay ${ }^{[4,5]}$, a micro-pits array with depth $6 \mu \mathrm{m}$ and diameter $14-16 \mu \mathrm{m}$ was obtained by Femtosecond laser texturing method. And the reflectivity of the surface is $20 \%$. Harvard University, professor Mazur also used femtosecond laser texturing method to get a spike structure on the surface of silicon wafer, visible light and near infrared wave were almost completely absorbed by the surface micro structure. Professor Mazur also formed that the surface micro structure play an important role to the light absorption rate. 
At home, Qiu M.B ${ }^{[6]}$ processed silicon based materials by means of Wire cut Electrical Discharge Machining (WEDM), obtained pit-cavity composite structure with $2 \mu \mathrm{m}$ embedded hole and 5 10 depth to diameter ratio of pits on the silicon surface. And the reflectivity of the surface is $5 \% \sim 6 \%$. Xiao J.F $\mathrm{F}^{[7,8]}$, Zhejiang University, gained a porous-pyramid composite structure on the surface of silicon.

\section{Analysis of surface topography in the process of electric discharge texturing (EDT)}

Single pulse discharge pit morphology. The morphology of the surface of EDT is mostly determined by three parameters of peak current, pulse width, pulse interval. At present, the relationship between electrical parameters and the surface topography is all derived based on the experimental dates.

According to the theory of thermal process, the volume of the discharge trace is ${ }^{[9]}$ :

$$
V=\frac{Q}{C \rho\left(T_{r}-T_{0}\right)+\rho q_{r}}
$$

In the formula:

$Q$ represents the total heat in the pulse discharge area, $\mathrm{J}$;

$C$ is specific heat, $\mathrm{J} /(\mathrm{Kg} \cdot \mathrm{K})$;

$\rho$ is density of the material, $\mathrm{Kg} / \mathrm{m}^{3}$;

$T_{r}$ is melting point, $\mathrm{K}$;

$T_{0}$ represents original temperature, $\mathrm{K}$;

$q_{r}$ is latent heat of fusion, $\mathrm{J} / \mathrm{Kg}$.

The pit shape is determined if the material on the surface is total melted when the temperature of pulse discharge area is higher than the melting point and the molten metal is total removed by the impact of torch and plasma. As shown in figure $1, R$ is the maximum pit radius, $H$ is pit depth, $S_{c}$ represents the pit ball radius. According to the sphere volume formula ${ }^{[10]}$, the volume of the single pulse discharge is:

$$
\begin{gathered}
S_{c}=H / 2+R^{2} / 2 H \\
V=\pi H^{2}\left(S_{c}-H / 3\right)
\end{gathered}
$$

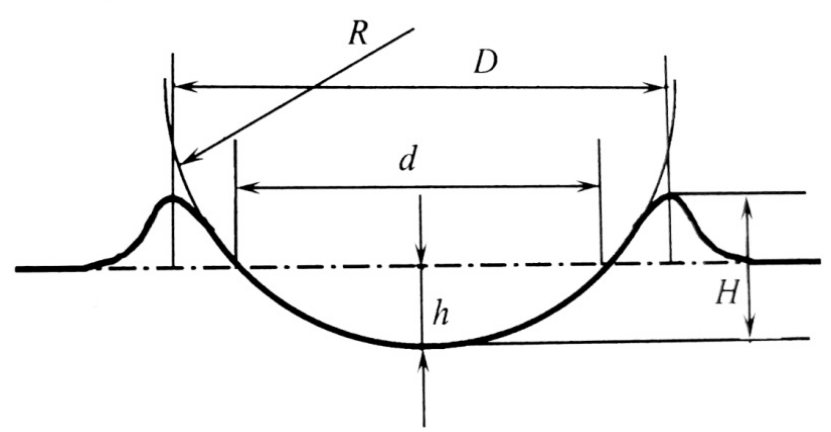

Fig.1 Schematic Cross-Section of EDM Hole

Under the condition of negative electrode, copper electrode, 10 1000 $\mu$ s pulse width and 27 144A peak current, Professor Fengguo Cao from Beijing Electric Processing Research Institute, used EDM to process steel, gained the relationship between the discharge trace size and electric parameters (pulse width and peak current). 


$$
\begin{aligned}
& h=(0.67 \sim 0.92) \times\left(\frac{\mathrm{K}_{\mathrm{g}}}{\pi \rho}\right)^{0.33} \times t_{o n}^{0.37} \times I p^{0.47} \\
& D=\mathrm{K}_{\mathrm{D}} t_{o n}^{0.4} I p^{0.5} \\
& M_{0}=\mathrm{K}_{m} t_{o n}^{1.1} I p^{1.1}
\end{aligned}
$$

In the formula:

$h$ is the depth of discharge pit, m;

$K_{g}$ is a constant, $\mathrm{Kg}=3.74 \times 10^{-4}$;

$\rho$ is the density of electrode, $\mathrm{Kg} / \mathrm{m}^{3}$;

$t_{\text {on }}$ represents pulse width, $\mu$;

$I_{p}$ represents peak current, A;

$D$ is measured discharge crater mean diameter, $\mathrm{mm}$;

$K_{D}$ is a constant, $\mathrm{KD}=9 \times 10^{-3}$;

$M_{0}$ is measured single pulse removal quantity, $\mathrm{g} /$ pulse;

$K_{m}$ a constant, $\mathrm{Km}=9.4 \times 10^{-11}$.

Known from formula(4),formula(5) and formula(6), single pulse removal quantity, the depth of discharge pit and discharge crater mean diameter three important parameters of pit all increases exponentially with the heat energy.

Morphology of multi pulse continuous discharge pits. Morphology of multi-pulse continuous discharge pits is formed by the generation and disorderly superposition of many single pulse discharge pits. When tool electrode being close to workpiece electrode and forming electric spark, surface protrusion was first to be breakdown by electric field and a channel between two electrodes is presented. So there is a big difference between multi-pulse continuous discharge pit morphology and single pulse discharge pit morphology.

$$
R a=\mathrm{K}_{\mathrm{R}} t_{\text {on }}^{0.3} I p^{0.4}
$$

In the formula:

$\mathrm{Ra}$ is measured surface roughness, $\mu$;

$\mathrm{KR}$ is a constant, $\mathrm{KR}=2.3$.

Influences of the electric parameters on the textured surface morphology

Influences of peak current on the textured surface morphology. When pulse width is $4.4 \mu \mathrm{s}$, peak current is $25 \mathrm{~A}, 33 \mathrm{~A}, 55 \mathrm{~A}, 60 \mathrm{~A}$, the micro topography (200 times) obtained from optical microscope are shown in Figure 2. 


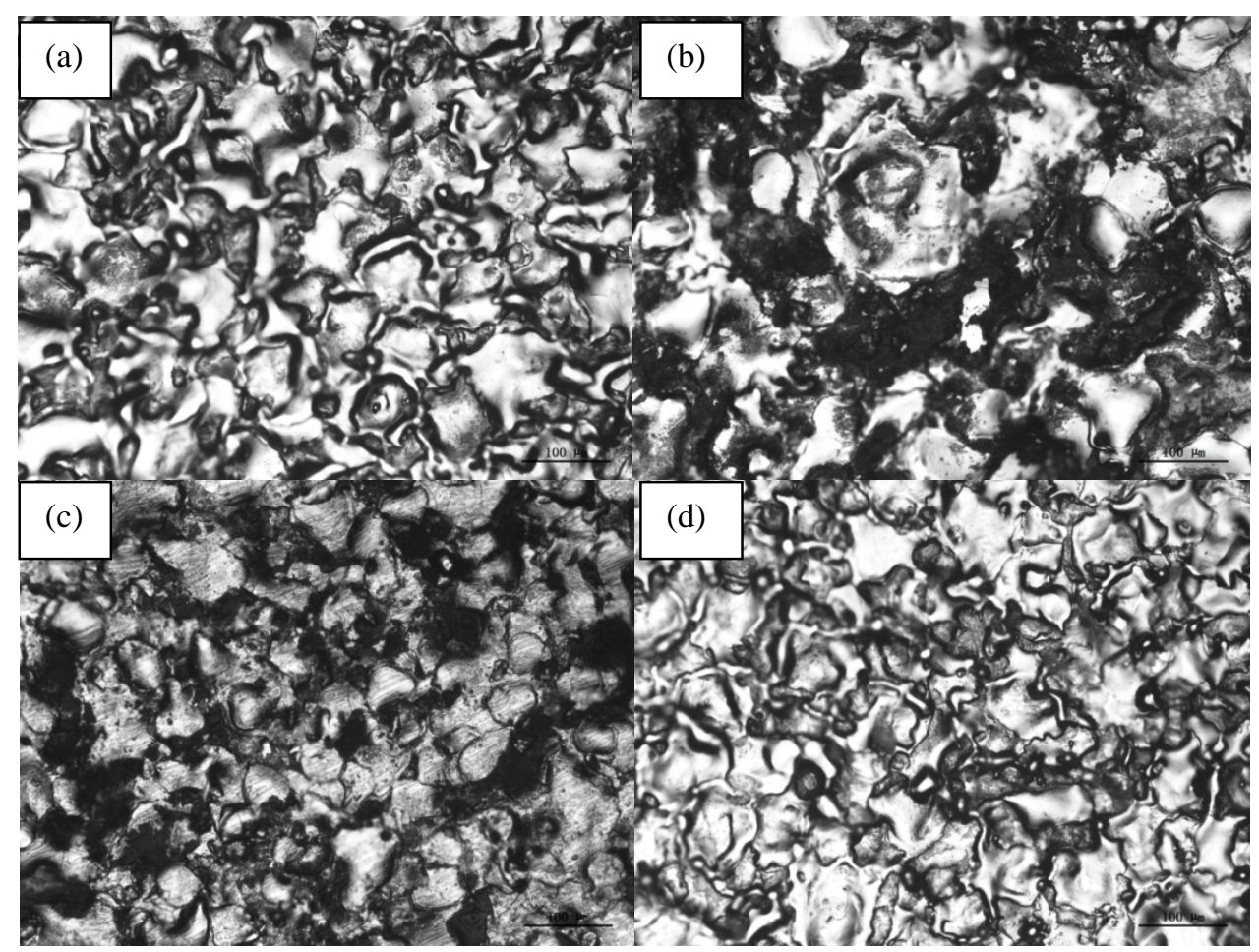

Fig. 2 The Microstructure by EDM $\left(T_{o n}=4.4 \mu \mathrm{s}, I_{p}=25 \mathrm{~A}(\mathrm{a}), 33 \mathrm{~A}(\mathrm{~b}), 55 \mathrm{~A}(\mathrm{c}), 60 \mathrm{~A}(\mathrm{~d})\right)$

As shown in Figure 3, with the increase of the peak current, the average diameter of the textured surface pit increases first and then decreases. From the microcosmic point of view, peak current determines the magnitude of the acceleration of electrons in the discharge channel and then affects the kinetic energy of the electron impact work piece. From the Macroscopic point of view, peak current affects the single pulse energy density. Peak current mainly affects the depth of the pit and the influence of peak current on the pit diameter is relatively small.

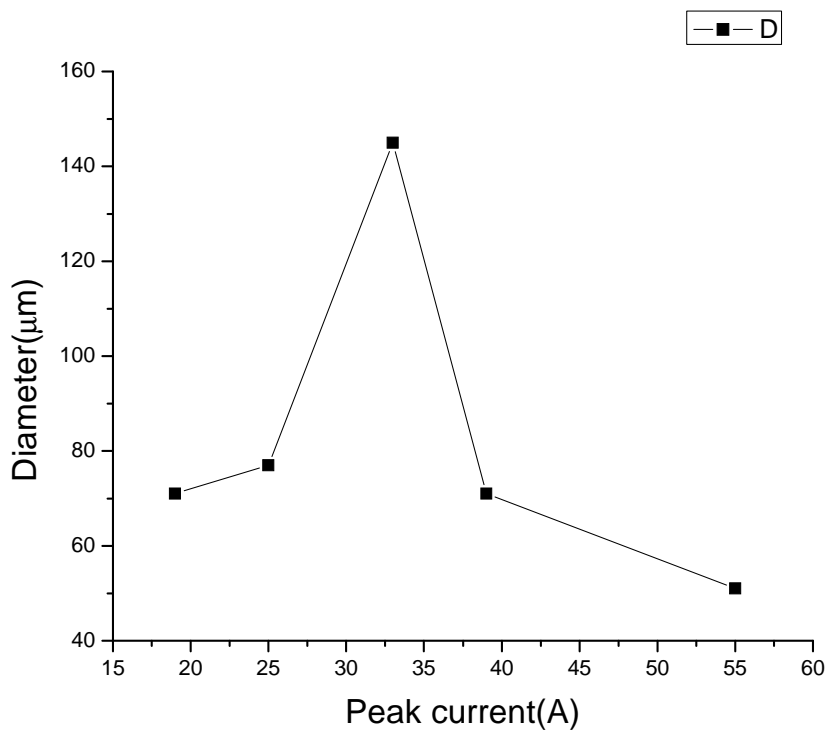

Fig.3 The Affection of Craters Diameter by Current

Influences of pulse width on the textured surface morphology. When peak current is $75 \mathrm{~A}$, pulse width is $25 \mu \mathrm{s}, 33 \mu \mathrm{s}, 55 \mu \mathrm{s}, 60 \mu \mathrm{s}$, the micro topography (200 times) obtained from optical microscope are shown in Figure 4. 

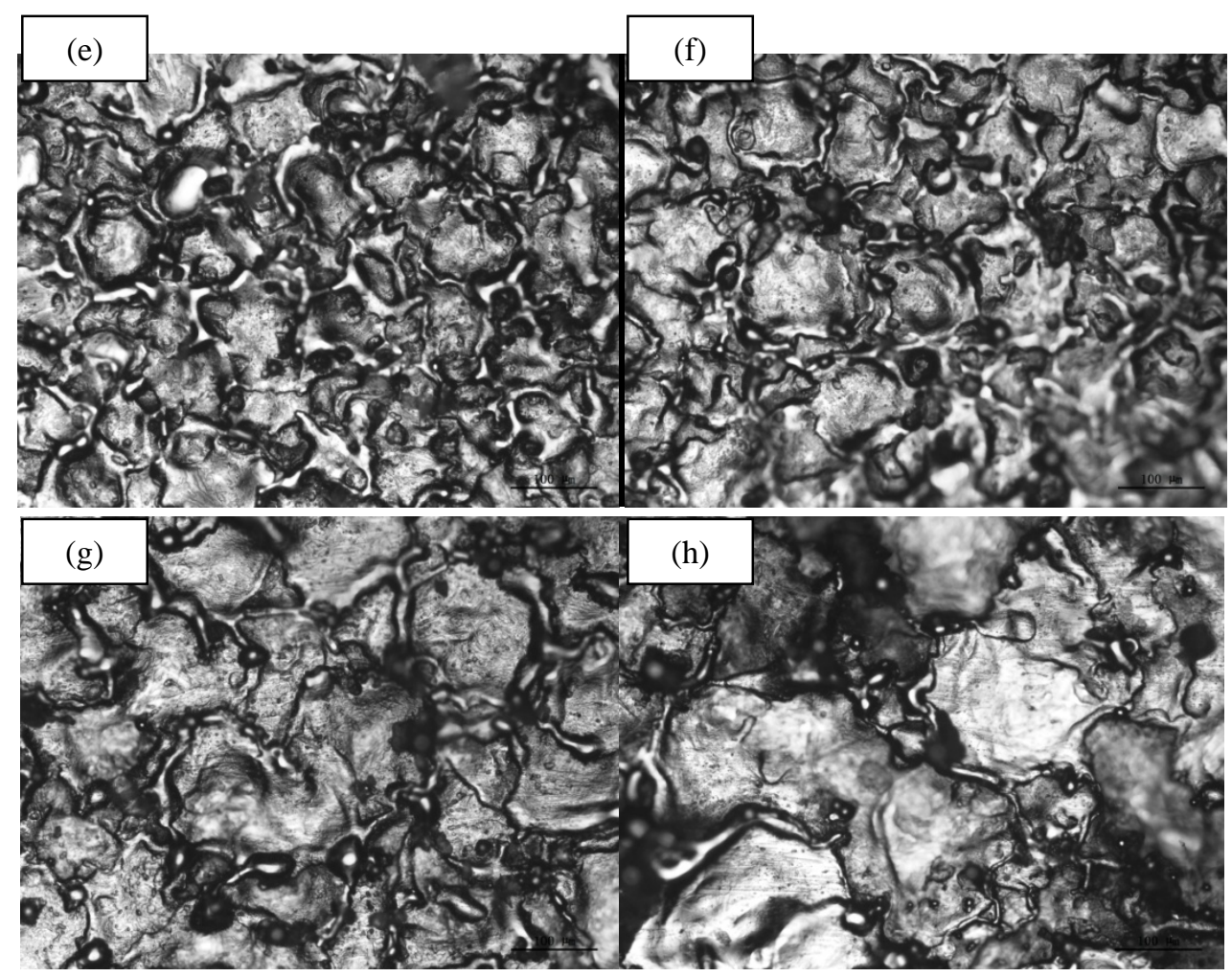

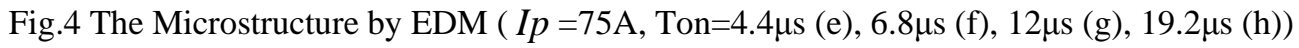

From Figure 5, the average diameter of the textured surface pit increase rapidly with the increase of the pulse width. From the microcosmic point of view, pulse width determines the magnitude of the ramp-up time of electrons in the discharge channel and then affects the quantity of the electron impact work piece. From the Macroscopic point of view, pulse width affects the time of single pulse and the machining area. Peak current mainly affects the average diameter of pit and the influence of pulse width on depth of pit is relatively small.

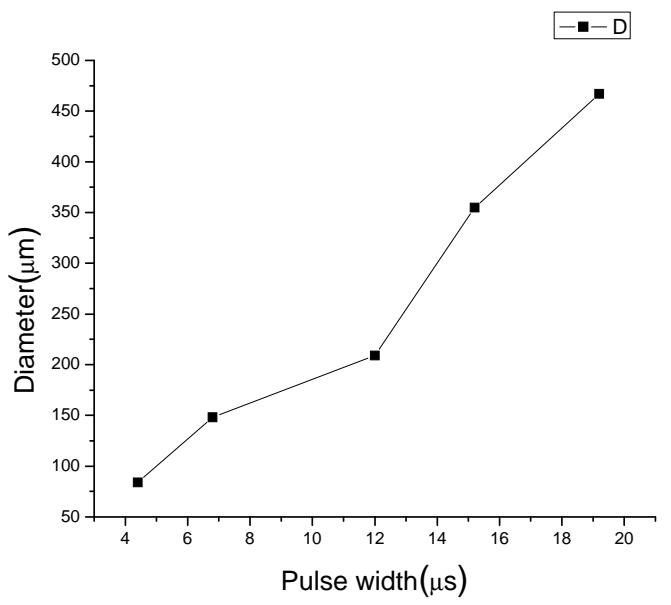

Fig.5 The Affection of of pulse width on Craters Diameter 


\section{Conclusions}

Experimental work has been performed to analyze the effect of the electrical parameters on the textured surface morphology of non magnetic steel (20Mn23AlV). It provided important quantitative results carried out on the selection of the machining parameters for obtaining possible high surface finish quality and machining outputs as follows: Two electrical parameters of peak current and pulse width have enormous implications on the structure of the pit. Peak current mainly affects the maximum depth of the pit and pulse width mainly affects the maximum diameter of the pit. Pulse width is the processing time of a single processing cycle, which mainly determines the number and density of electrons to hit the workpiece surface, and then influences the size of the hot melt area. Peak current determines the rate of electrons and impact force on the surface of the workpiece, and then influences the machining depth.

\section{Acknowledgements}

The work is supported by the Science and Technology Department of Jiangxi Province (Item No.: 20142BBE50066).Authors would like to thank the tutor for the help in language, kind encouragement and technical support.

\section{References}

[1] Murray,Sun,Patil, Wood,Clare,Physical and electrical characteristics of EDM debris, Journal of Materials Processing Technology,2016.Vol.229,54-60.

[2] R. Carsten, L. Falk. Photon Management by Metallic Nan-Odiscs in thin film solar cells [J].Appl. Phys. Lett, 2009(94):213102-1-3.

[3] J. Terpák, L. Dorčák, J. Revaj. Quality Control of the Electronic-Discharge Texturing [J].Ctoatian Metallurgical society, 2010(49):01-06.

[4] L.A.Dobranski, A.Drygala, K.Golombek. etal Laser surface treatment of mc Si for enhancing optical properties [J]. Journal Mater. Process Technol, 2008, 201:291-296.

[5] U. Gangopadhyay, S.K. Dhungel, P.K.Basu etal. Comparative study of different approaches of multicrystalline sillicon texturing for solar cell fabrication [J] Solar energy mater sol. cells, 2007, Vol. 91(4):285-289.

[6] Qiu M.B,HuangY.H,Liu Z.D, A numerical study of the light reflection effect of silicon surface morphology[J], Journal of Optics, 2008, Vol. 28(12): 2394-2398.

[7] Xiao J.F, Study on the surface trapping structure of crystalline silicon solar cells[D],Zhejiang Master Dissertation of Zhejiang University, 2010:15-18.

[8] Zou S.F, Zhang T.,Surface vitrification of Fe36Co36Si4.8B19.2Nb4 alloy and its properties by eletrical discharge machining, Functional material,2016.9(47),09149 09152.

[9] Xue L, Theoretical analysis and test research on discharge gap of spark discharge channel[D], Shanghai: Master Dissertation of Shanghai Jiao Tong University,2011.

[10] Wu C.C,Zhang J,Chen X.L, EDT roll surface pit forming process[J], Journal of University of Science and Technology Beijing, 2007, Vol. 29(01): 59- 62. 